\title{
News Release System
}

\author{
Ping Wang \\ College of Information Engineering, Jiangxi University of Technology, Jiangxi Nanchang
}

\section{Keywords: Global informatization; Timely; Electronic commerce}

\begin{abstract}
With the development of society and progress of information technology, Internet has become more and more popular with an even closer relationship with people's life. Meanwhile, the trend of global informatization has also become increasingly obvious. It has become part of people's daily life to browse news quickly and acquire colorful information from the Internet. Thus, any large enterprise should take active part in global market competition instead of being restricted to one certain area. This news release and management system has conformed to current development, which not only saves valuable manpower and material resources, but also embodies the timeliness of news itself.
\end{abstract}

\section{Introduction}

During the global competition, the enterprise's competitiveness will be greatly measured by its mastery of information, acquisition of information, employment of information as well as reaction to information. At the same time, more and more enterprises have established their own websites so as to display their products, release the latest news and communicate with their customers, and thus they can establish partnership with them to conduct electronic business and so on, within which news administration system is an important part of enterprise website with double effects. On one hand, it can be used to release related new products or new development events dynamically; on the other hand, operational performance, technology and development progress as well as some special or preferential projects, products and services in the enterprise can be announced to customers through it. By this way, customers can be attracted and enlarged. As is known to all, our society is full of information. Large amount of manpower and material resources are needed to collect, release and update information every day. In the new age of Internet development, more and more information has been released on the Internet, and it's no exception for news, one important subject of information. Generally, there are two ways of Internet news management. One is static HTML page, when updating information, corresponding pages should be reproduced and uploaded with the modification of corresponding links. However, with the further development of Internet, development and maintenance of such static website has become more and more difficult since large quantity of information costs huge manpower and material resources if they are produced one by one through static website simply; besides, the timeliness of news itself can't be brought into play without any value. What's worse, the static website can't be interacted with browsers effectively, so this method has been abandoned gradually. Another method is to combine dynamic website with database based on ASP, PHP, JSP or scripting language so as to processing information by application programs, which is quite popular at present. This news release and management system 


\section{Analysis on System demand}

The news release system, which is also called information release system, is a website application program managing certain variable Internet information collectively such as news, releasing of new products as well as dynamic business news; besides, the information will be classified by their common properties. At last, they will be released on the Internet systematically. The Internet information is added to a database through an easy interface, and then it is released to website with existed webpage template as well as review process based on web application program of web browser.

Research on feasibility. Technical feasibility:

The whole system is of simple development since it is developed and operated with the employment of JSP, Dreamweaver, Tomcat and MYSQL; besides, software like Dreamweaver and MYSQL can be operated under graphical interfaces, which has further lowered the complication technically. As for the establishment and management of database, they are obtained by simple addition, removal, locating and edition, so the difficulty can be overcome completely. In addition, some deployment of environmental variables can be installed for the software with addition of JDBC, the driving device, to corresponding folder of Tomcat. Moreover, lots of related documents and courses can be referred to. Therefore, the system is simple for its installation, configuration as well as final development and operation.

Economic feasibility

As for any system, it costs relatively more money in the early stage, but the afterward expenditure is comparatively less since the system itself occupies less space so that it can be coexisted with other systems in the same database. Due to dynamic webpage design, update of news becomes simple and convenient without much manpower and material resources, which can improve administrator's working efficiency with lower cost. Therefore, it has relatively greater economic advantages.

Feasibility of the usage

This system has simple operation, beautiful and effective interface with addition of much humanized design, which makes the usage more convenient and efficient. By simple click, readers can receive necessary news information they need. Besides, news has been classified into different varieties by individualized need so that readers can locate the news they need quickly with a larger reading space. In addition, the black-stage management is even easier since the visualized operation of database makes things easier, and the usage of HTML online editor has also brought much convenience for news update. All in all, this system is very easy to operate.

Analysis on functional demand. The interface of front-desk service. It includes design of beginning and ending on the front page with three-class column, covering pictures as well as hypertext links, etc. In terms of the news display, both the display of single news and related news should be contained with some comments so that readers can locate the news they need clearly and easily. The news should be classified strictly by their contents with addition of relative remarks and comments. Meanwhile news search as well as keyword search should be supported.

The interface of back-stage management. Limits to logging in for administrator's account and member's account should be distinguished. Administrator should have the right to manage users as well as update, modify and delete news, which covers first-class column management, second-class column management and third-class column management. Addition of news should be be convenient and fast with management and maintenance of related comments. 
Analysis on the functions of the system. Design idea of the system. Pages should be modularized with flexible deployment of webpages. Meanwhile, the back-stage maintenance and the front-stage display should be separated.

Division of functional modules of the system. This part can be expressed by two figures below as Fig. 1 and Fig. 2.

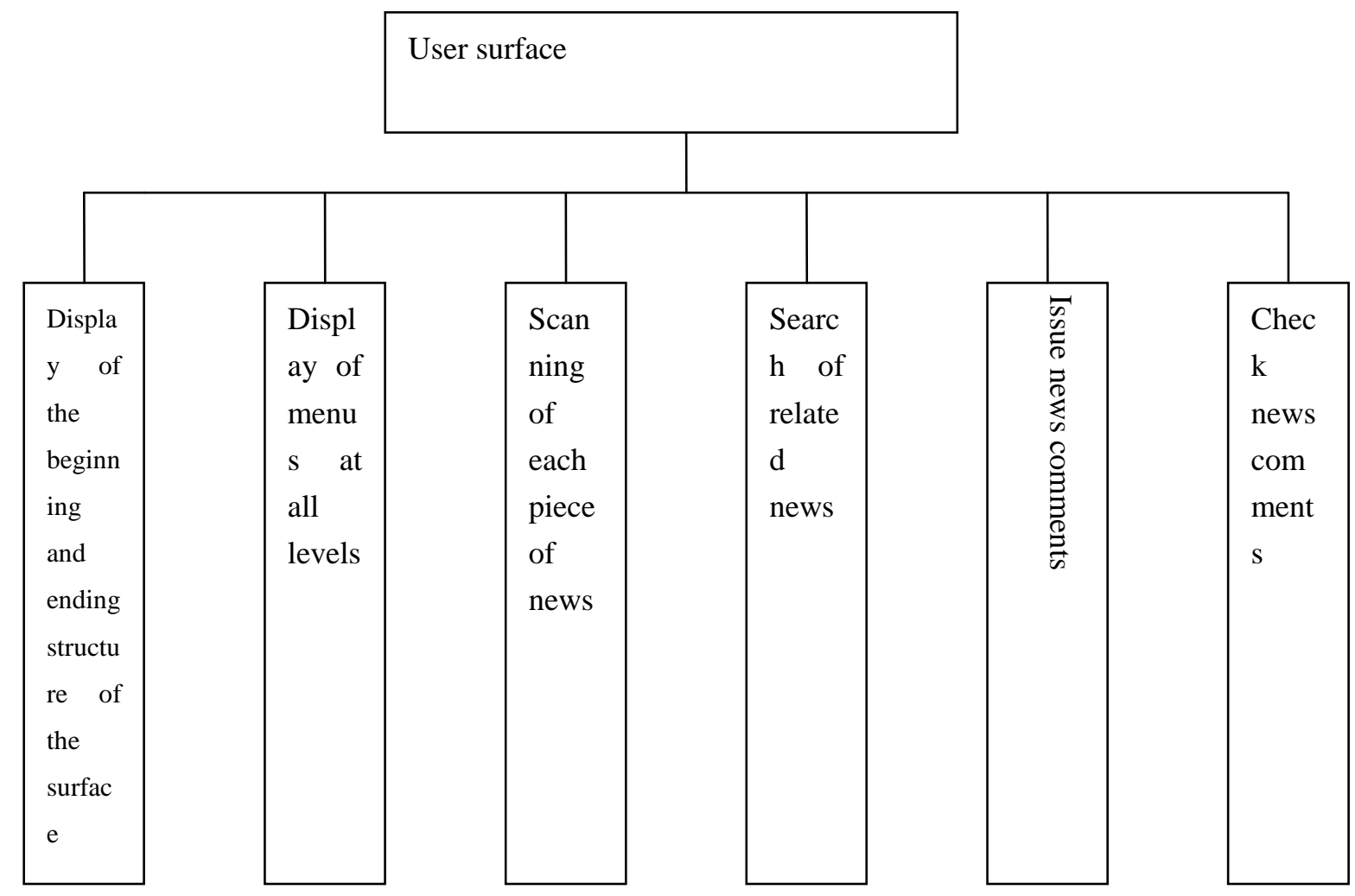

Fig. 1 Functional module diagram of the system for front-stage user surface

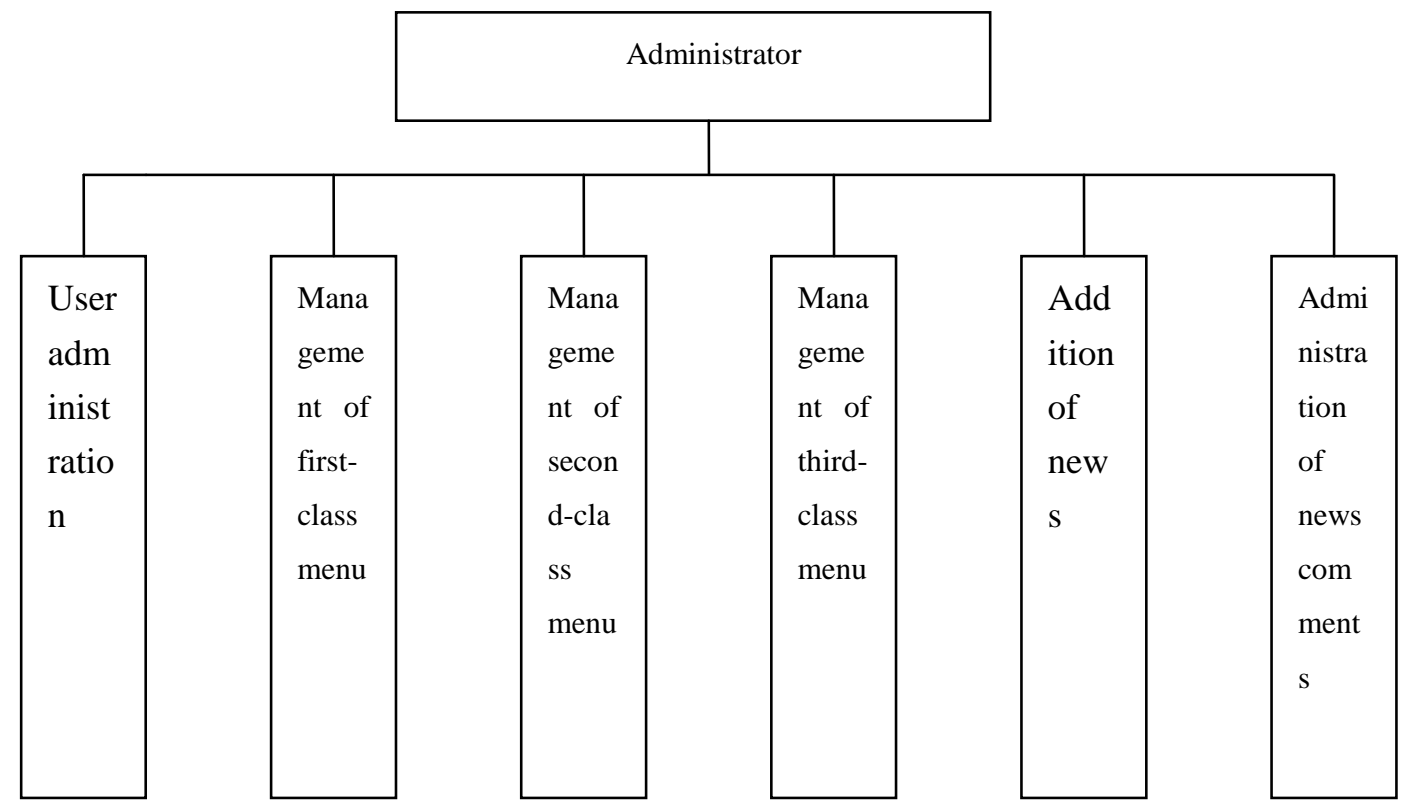

Fig. 2 Functional module diagram of the back-stage management surface 
Structure design of the system. The following Fig. 3 can be drawn according to systematic functions and characteristic summarized above

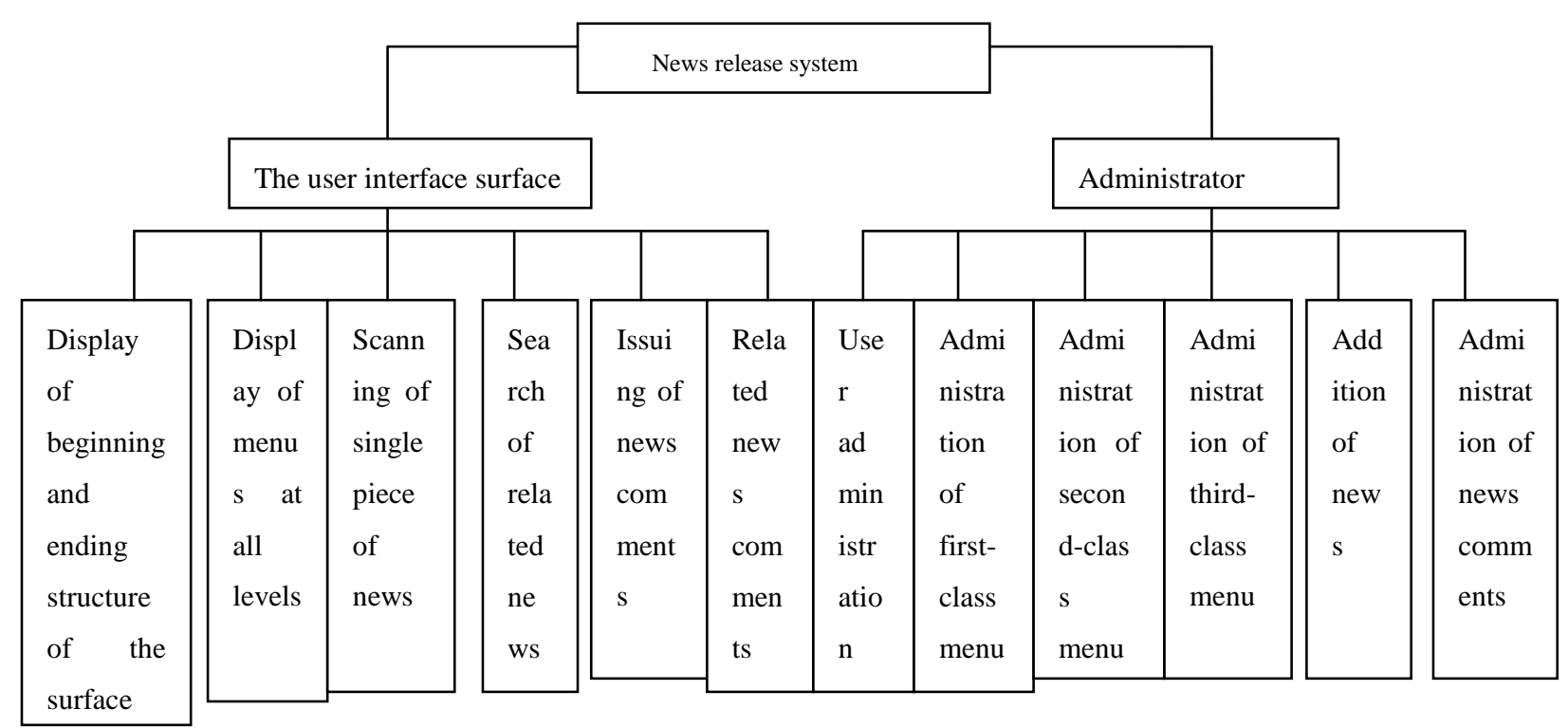

Fig. 3 Structural design diagram of the system

\section{Design of Database}

Design of database. The design of database is a customer-oriented logic design, which classifies and groups data by customer's need from the perspective of logical level. As for the design of database, archived data of each department as well as their demand for data should be considered together with analysis on the relationship among various data so as to design a data model according to functions and descriptions offered by DBMS, which can reflect the relationship correctly and meet various inquiry needs with appropriate size, less data redundancy, high access efficiency.

Analysis on the demands of database. Factors from two aspects should be taken into consideration when analyzing the demands of database since this system is oriented to two kinds of objects, that is, users and administrator.

As for users, what they care about are scanning, search and comments of news; and meanwhile they also pay attention to column path information, information of news pictures, recommended news information, click information of news and audit information of news, etc.

Through systematic analysis on the functions, demand information has been summarized as follows addressing common users of news system.

Each column should have a corresponding folder.

Each piece of news should have a corresponding JSP folder.

Each piece of news can only be grouped into one column.

There should be an article under the child column of each piece of news.

One column can have many pieces of news.

Same keywords can be used for news under different columns.

Each piece of news can't be accessed to users before auditing.

Beside basic information, each piece of news should also include path information, information of pictures, recommended news, comments as well as clicks and so on. 
As for administrators, what they care about are addition, audit, modification and deletion of columns and news. Different administrators should have different privileges, which can be divided into three classes. The first class is senior administrator who can do anything he wants; the second class is audit administrator who can add and audit news; the third class is clerk who is only responsible for typing in the news. According to analysis above, the following demand information has been summarized for system administrators.

Different administrators have different privileges.

Administrators can give addition, deletion and modification to each class.

Addition, deletion and modification can be made by administrators to each level of column.

Administrators can add, delete or modify news.

Administrators can add, delete and modify the comments of news.

Administrators have the right to audit news.

Administrators can administrate users.

After analyzing functions of database system and summarizing the demands with consideration to future functional expansion, data item and data structure as follows have been designed.

Information about administrator covers the name, password as well as privileges of the user.

Information about the column should cover the serial number, title and path of the column and so on.

Information of the column covers the information of column title, coordinate position of hot spots, linking address, second-level column and pictures.

As for news information, its data items cover news number, column, news title, news content, news pictures, audit information, source of information, news author as well as name of the folder and so on.

Design of a conceptual structure for the database. Withe the data items and data structure gained above, we can lay a solid basis for later logic design by designing various entities as well as relations among them to satisfy users' needs. These entities include various concrete information, which forms data flow with mutual interaction. Specific designed and planned entities are administrator information, entity of column information, news entity and entity of user information.

\section{Design of the System}

Compiling of components. Like a tall building, system is not established in a minute. Components in system or program are just as bricks which constitute a whole system. Luckily, components are relatively independent, which can be employed directly without repeated compiling. Due to this characteristic, the work load of programming has also been reduced.

Compiling of Bean for string processing

One important component in a system is character string processing Bean, which is a function. As a commonly used character string processing, it mainly deals with input and output of character string since data used by general programs is expressed in character strings. It is very important for a system to have the functions of displaying numbers and time correctly with good processing of character code, which raises different demands for different systems.

Compiling of user administration Bean

This function is used to conduct maintenance for back-stage system users. The back-stage administrative programs can only be used after authentication by users. Thus, the function of maintenance should be offered to users by back-stage necessarily. 
The system administrator who can add, modify, delete, hang up and restore other users is the default user of the highest-level during program initialization. Only legalized users have the right to $\log$ in back-stage system. Functions offered by this folder include addition of users, modification of user property and deletion of users and so on.

Compiling of maintenance Bean for columns of various levels

This function is used to maintain column titles of different levels in the front-stage of the system. The function of add () will be used when adding columns, the function of mod () will be used when modifying columns, and the function of del () will be employed when deleting columns. Before employing functions, the function of set ID () should be used first to set the following operation for specific column.

Besides, this set of components supply the function of inquiry. If related properties of a certain column need to be inquired, the function of getOneData () needs to be employed. If all column lists need to be inquired, then the function of getOnePage () shall be used. This function is a page function, which is responsible for offering the data of particular pages and accepting parameter setting of lines on each page.

News release and compiling of search Bean

This function is used to maintain news in the front-stage of the system. The function of add () is used to add news, the function of mod () is used to modify the property of news, and the function of del () is used to delete news. Before employing the functions, the function of setID () should be used to set operations for following news.

In addition, this set of components has also provided the function of inquiry. If related properties of a certain column or all column lists need to be inquired, then the same functions should be employed as before. These functions can also provide data for particular pages and accept parameter setting of lines on each page.

This set of components also has the function of inquiring on homepage. Type in keyword by employing the function of search (), the system can return to the list of article titles.

A detailed design of the system. After discussing functions of each component as well as the calling methods, let's have a discussion about the front and back stages of the system, which are truly used by users. Just now, components are compared to bricks, but front and back stages are just as the inside and outside walls of the whole building since they are exposed to users directly. Speaking of usage, it can not live without management. Next we'll discuss the management surface of back stage first.

Design of the management surface for back stage

The model design for the back-stage user administration is addressing maintainers. There is a default back-stage administrator of the system, who is responsible for establishing new system users after users logging in the back-stage administration model. If the new user is in a "working condition", then it means that the new user can log in and use the back-stage system; however, if the new user is in a "hanging up" condition, then he or she can't log in successfully until the condition is modified by system administrator. The purpose of such design is to avoid unnecessary troubles. For example, if there are five users existed, but some of them are wanted to stop using the system in a while, then their identities will be hung up by retaining the logging information so that they can be recovered directly without establishing new accounts. For sure, administrator of the system can also delete users. Information displayed on the surface contains user name, password, condition and 
remarks. The user can only be valid in working condition, and if it is shown hanging-up condition, then it indicates this user can't log in the back stage for administrative operations.

The news release module is the main module of the system, which is used to maintain news contents in the front stage. The foundation of this module is the three modules introduced above, which can add, modify and delete news. Its field information includes titles, levels of column, affiliated columns, news titles, news sub-titles, keywords, name of the folders, audit and approval, author of the news, news journalist, source of news, operator, time of release, time of modification and contents of article and so on. As for the edition of news contents, the system realizes it with a editor of script news contents. Characters can be edited in the text box with adjustment of the format, and meanwhile pictures can be pasted so as to reach a visual effect conforming with the homepage.

Comments on the news are released and displayed on the front stage, but they can only be deleted by administrator on the back stage. This function is used by administrator to administrate comments issued by users on the front stage, for example, the administrator can check and delete the comments. Since each piece of news supports the function of comment, there will be backlog data for comments in a long term. Therefore, they should be dealt with and cleared regularly.

Design of customer surface on the front stage

As is mentioned above, the front stage is just as a complete presentation layer of the system. Both design and maintenance of the back stage serve for front stage. In another word, they are operated by the front-stage needs. Thus, it is especially important to front stage. Likewise, there are three classes of columns for front stage, but they don't have the inclusion relations. Such kind of design is really flexible and convenient for satisfying actual needs of customers.

The first-class column should be located in the left part of the homepage. The column icon here is designed into a complete picture for unified administration. Therefore, when reading database information, the actual position of the mouse should be correlated with relative properties of columns. Since the position of click has indicated current coordinate position of mouse, users are wanted to fill in the accurate position of the mouse during the design of the first-class back-stage column. The defined position information read from database will be shown here. Then if we define the hot spot of mouse into this coordinate, then the first-class column can be manifested correctly.

The contents of news information display on the main section. The abstracted contents are edited by administrator in the back stage when releasing news. In a piece of news, text, pictures and hyperlinks are basically found. When editing the news, administrator can adjust and express the contents accordingly for a better outlook.

Related news can be searched by related keywords set during the news releasing. By offering related keywords, the system database can help users to find all news articles by blur match. Besides, the searched news lists will be displayed in the news section so that users can check the detailed contents of the articles by clicking the news title.

As for the part of news comments, it is prepared for users to issue comments if they have some ideas while scanning some articles. What they need to do is click the button of "post a comment". Information on the form page including the releasing time, IP the commentator, comment contents and so on. The comment contents will be stored in the database after being submitted, and they can be viewed by other users. 


\section{Conclusions}

The surface should be made concise, decent and serious as it is news release system, and it should offer convenient and fast to users as possible it can. The development of news release system should be combined with the design of back-stage database. The system established this time still incomplete with some functional limitations, which needs to be further perfected. As the basis of information release system, an excellent design of database can make the system more powerful regarding functions with better employment of information, the valuable resource. The connection of database is also the most important section of the whole design. First, a flow chart of the system should be drawn, and then an information table of database should be designed as well as all information covered, which requires a full consideration. In this way, it's unnecessary to add some new information tables after completing the system. With the final completed news release system, all functions related to news scanning, message board as well as online survey can be realized. In the module of online survey, percentages of options can even be counted so as to give illustrations intuitively.

\section{Acknowledgements}

This work was financially supported by project of Technology Department of Jiangxi Province [No 20143BBM26048] and the project of Technology Department of Jiangxi Province [No 2013BBE50051] also gives us lots of help.

\section{References}

[1] MS SELINA CHA. Development and Application of Electronic System Navigation. First Edition. Beijing: Posts \& Telecom Press, 2004: P1-3.

[2] Yu Xiangdong. Brief Analysis on the E-government Development At Home and Abroad. Information Technology \& Standardization, 2003. Third edition: P14-15

[3] Wang Xiaoyi, Liu Zhongbing, Xu Jiajing etc. Excellent Examples of JSP Database Development. The first edition. Beijing: Publishing House of Electronics Industry, 2005: P9-10

[4] Robert J Brunner, Frank Cohen.Java web services unleashed[M].2002: P32-34

[5] Sa Shixuan, Wang Shan. An Introduction to Database System [M]. Third Edition. Beijing: Higher Education Press, 2002: P4-5

[6] Zhang Haifan. Software Engineering[M]. The first edition. Beijing: Posts \& Telecom Press, 2002:P91-92. 